\title{
HOMEWORKING: ESTRATEGIAS COMUNICACIONALES Y SENTIDO DE PERTENENCIA
}

\author{
AUTOR: COELHO, Nicolas Joel \\ Profesor - Universidad Nacional de Córdoba - Argentina \\ nij.coelho@gmail.com
}

\section{Resumen}

los beneficios comunicacionales y operativos de las TIC para alcanzar objetivos organizacionales son claramente conocidos, pero, a la hora de transmitir valores institucionales, ¿qué cambios generaron? En la siguiente investigación se analizará cómo el nuevo método de trabajo homeworking (que se basa en el uso de las TIC) ha introducido cambios en la planificación de estrategias comunicacionales en lo que refiere al "sentido de pertenencia" que tienen los empleados hacia su institución, pensando en ¿cómo se transmite este sentido de pertenencia en los métodos de trabajo donde todas la relaciones son mediadas por las TIC?

Para responder la pregunta, se realizó un estudio de caso a una empresa radicada en la ciudad de Córdoba, Argentina, a partir de un diseño metodológico exploratorio y descriptivo, empleando las técnicas de la entrevista y el análisis de contenido para su abordaje. Una vez realizado el trabajo, las conclusiones a que se llegaron confirman que, a pesar de las posibilidades que las TIC ofrecen en materia comunicacional, el medio más importante a la hora de transmitir valores sigue siendo la relación cara a cara. Con esta investigación se busca un nuevo aporte a los estudios de comunicación institucional en un fenómeno que, por tendencia, tiene un constante crecimiento global.

\section{Palabras Clave}

Comunicación directa; comunicación mediada; cultura organizacional; estrategias comunicacionales; homeworking; sentido de pertenencia, TIC.

\section{Introducción}

La comunicación está condicionada en la organización por una serie de cambios y mutaciones que afectan la estructura social, lo que está influyendo en la aparición de nuevas modalidades de organización y de cultura del trabajo (García Jiménez, 1998). Una de esas nuevas formas de organización es el método de trabajo homeworking. Luego de indagaciones y distintos aportes, el homeworking fue definido en el estudio como un método para realizar una actividad laboral en relación de dependencia, fuera del espacio físico de la empresa (oficinas), con autonomía de trabajo, y que se genera a través de las nuevas Tecnologías de la Información y la comunicación (TIC).
En Argentina, la atención prestada a esta forma de trabajo comenzó a partir del año 2002, a causa de la severa crisis económica con altos niveles de desempleo. "Trabajar desde casa" a través de las TIC empezó a verse como una oportunidad única para responder a una situación que dejaba escasas alternativas.

En ese momento, era incierto el número total de trabajadores que se desempeñaban como homeworkers. Sin embargo, Carrier y Asociados ${ }^{1}$,

\footnotetext{
${ }^{1}$ Estudio profesional dedicado a la información y análisis de mercado con el foco puesto en el consumo y uso de productos y servicios vinculados a Internet, las telecomunicaciones, la informática y los medios digitales.
} 
analistas de mercado, publicó los resultados de una encuesta sobre "Telecomunicaciones residenciales"; en la cual exhibía que, para 2003, había más de 320.000 hogares usados como entornos laborales electrónicos y representaban el $3.2 \%$ de todos los hogares del país.

Ya para 2013, la doctora Viviana Laura Díaz, coordinadora a cargo del área de teletrabajo del Ministerio de Trabajo, Empleo y Seguridad Social, afirmaba que: "Actualmente, el teletrabajo está creciendo de manera agigantada en nuestro país, calculándose más de 1.800.000 oficinas en el hogar" (Bellomo, A. (2013). "En nuestro país el teletrabajo está creciendo de manera agigantada, calculándose más de 1800000 oficinas en el hogar". Recuperado enero 15, 2013, de Eleve Sitio web:
http://www.ele-ve.com.ar/En-nuestro-pais-elteletrabajo-esta-creciendo-de-manera-agigantadacalculandose-mas-de-1800000-oficinas-en-elhogar.html)

Así, año tras año se ha ido produciendo una transformación del trabajo prototípico en las organizaciones, es decir, aquel que se desarrolla a tiempo completo y dentro de las dependencias de las mismas. Las nuevas tecnologías y en especial las de la comunicación, han ayudado a potenciar estos nuevos conceptos, originando nuevas concepciones de empresa y de sus estructuras laborales.

Estos cambios en los métodos de trabajo y la influencia de la gestión de comunicación son el objeto de la actual investigación, enmarcada en un estudio de caso.

\section{Cultura, sentido de pertenencia, afiliación y comunicación}

La dimensión de la cultura organizacional referida al sentido de pertenencia cobra una especial importancia modificando los modelos clásicos conocidos. Al respecto, por sentido de pertenencia se entiende a la consolidación de la adaptación y el incremento significativo del nivel de fidelidad, implicación y compromiso para con la organización, es decir, cuando los públicos internos personalizan en sus comportamientos, desempeño y discursos el proyecto institucional explícito en la misión, visión y valores de la empresa (Álvarez Nobell et al., 2012).

La importancia que presenta para las empresas contar con empleados de marcado sentido de pertenencia es fundamental. Permite generar ambientes positivos, resolver problemas eficientemente, alcanzar objetivos, mejorar servicios, ahorrar capitales, etc. (García Jiménez, 1998). Se afirma así la relevancia de la gestión de los recursos humanos en todas sus formas $y$, específicamente, la importancia de contar con empleados que corporicen los valores institucionales (Scheinson, 2009).

Otro concepto de importancia es el de afiliación. El mismo ayudará a entender que, en muchos casos, la idea de pertenencia leal, sólida y duradera de años anteriores a una organización no puede desarrollarse ni sostenerse actualmente. Por afiliación se entiende la función psicológica que cumplen los grupos, relacionada con la necesidad de amistad, apoyo moral y afecto por parte de los individuos (Schein, 1982). En otras palabras, la afiliación a un grupo parte de la necesidad psicológica de los individuos de formar relaciones sociales, lazos de amistad, identificaciones y sustento moral por parte de un grupo. Necesidad de formar parte, sentirse querido por los pares y formar lazos que los afilien.

Los grupos son, ante todo, un medio para satisfacer la necesidad de afiliación. El prototipo original de estos grupos, conocido a menudo como "grupo primario" es, desde luego, la familia. Todavía en la edad adulta dependemos de la familia para satisfacer estas necesidades pero también se necesitan grupos de amigos, grupos de trabajo y otro tipo de relaciones para satisfacer las necesidades de afiliación (Schein, 1982).

Siguiendo esta lógica, y relacionando lo anterior con la idea de sentido de pertenencia, se incorporará este concepto al estudio y se concluye finalmente que (para esta investigación) el sentimiento de pertenecer a una organización será poder reconocer su cultura, alinearse con la misma, entenderla y lograr que la persona logre un cierto afecto, una amistad con su grupo de trabajo e institución, que sienta afiliación a la misma. 
Entendiendo esto, se plantea así el escenario para analizar las estrategias comunicacionales en los nuevos métodos de trabajo, influenciados por los cambios tecnológicos, para generar sentido de pertenencia en los empleados.

\section{Contribuciones}

$\mathrm{Si}$ bien no existen precedentes que hablen específicamente del mismo tema, se destaca la existencia de abundante bibliografía en español e inglés relacionada a los conceptos de teletrabajo, grupos geográficamente dispersos, grupos virtuales de trabajo, comunicación institucional, trabajo con TIC y tecnología de la información específicamente, los cuales se relacionan con los fundamentos del presente estudio.

Como primer antecedente, en el año 2003 la Licenciada en sociología y Doctora en Ciencias Sociales, Paula Lenguita (2005), realizó en el Ministerio de trabajo de la Provincia de Buenos Aires (Argentina), una presentación acerca del "teletrabajo y el impacto de las tecnologías informáticas y comunicaciones sobre el empleo". En la misma se menciona cómo las Tecnologías de Información y Comunicaciones (TIC) son parte importante del presente comunicacional en las organizaciones y cómo el teletrabajo ha ido ganando terreno en el campo laboral, convirtiéndose en un eje más a contemplar en la discusión sobre las políticas públicas en materia de trabajo y empleo.

Por su parte, entre los autores que tratan el tema del homeworking y la comunicación interna, destacamos el texto de la Doctora Martha Alles (1999) titulado "Empleo: discriminación, teletrabajo y otras temáticas”, como así también el trabajo sobre comunicación interna del Doctor en filosofía y letras Jesús García Jiménez (1998) que se titula " $L a$ Comunicación Interna”. En los mismos se destacan los conceptos relacionados con los estudios en comunicación institucional y su relación con las TIC, brindando una guía completa y compleja de herramientas teóricas para el abordaje de cualquier institución desde el campo comunicacional.

Otro antecedente importante es el aportado por Antonio Pasquali (1970) en su libro "Comprender la
Comunicación" donde se reflexiona acerca de la conceptualización multidimensional de la comunicación. También resultan de interés los aportes de Manuel Castells (2009) en "La era de la Información. La sociedad de red”, quien se centra en los efectos sociales de las tecnologías de la información, mostrándonos los principios y avances de las nuevas tecnologías, siendo la información la materia prima sobre la que actúan las mismas.

Por último, se puntualiza un compendio de investigaciones que une a varios autores norteamericanos, a saber: Sessa Valerie I., Hansen Michael C., Prestridge Sonya y Kossler Michael E. (1999). En este libro titulado "Geographically Dispersed Teams: an annotated bibliography", los autores dan un giro al fenómeno y definen a los trabajadores de esta modalidad como Grupos Geográficamente Dispersos (por su siglas en inglés se los nombra como GDT's). En el mismo, los GDT's son caracterizados y se propone una forma de análisis desde las variables de tiempo y espacio, pudiendo éstas coincidir o no entre los integrantes de un mismo grupo. En otras palabras, se habla ahora de grupos de trabajo, no de trabajadores individualizados, donde sus miembros podrán estar en mismo tiempo pero diferente espacio, mismo espacio pero diferente tiempo, o diferente tiempo y diferente espacio.

De esta manera, concibiendo la diversidad de aportes desde diferentes campos que permiten dar una base de entendimiento al estudio, se buscará dar cuenta de cuáles son las estrategias comunicacionales que permiten desarrollar el sentido de pertenencia en trabajadores bajo el método homeworking, estudiando el caso de la empresa Nexius de la ciudad de Córdoba, Argentina. 


\section{Acercamiento a Nexius Córdoba}

Nexius es una empresa líder en servicios inalámbricos y soluciones de software para las industrias de todo el mundo, originalmente fundada en 2001.

En nuestro país y, específicamente, en Córdoba, los orígenes de Nexius se remiten al año 2003. En esos años, comenzó a prestar servicios básicos de soporte logístico en administración y contabilidad. Sus tareas se limitaban en asistir a los directores de empresa con todo lo relacionado a facturación de la misma.

Para el año 2005, esta sede contaba con seis personas que realizaban dichas tareas desde Córdoba para Estados Unidos: esto involucraba viajes continuos por parte de los miembros de Nexius Córdoba, como así también por parte del Presidente y director ejecutivo de la empresa en los Estados Unidos hacia Argentina, principalmente, con el fin de capacitarlos, tanto en herramientas de trabajo como en la filosofía organizacional de Nexius.

A fines del 2006, Nexius plantea la necesidad de establecer un equipo de desarrolladores de software en Córdoba. Así, para enero de 2007, se convocó a un grupo de profesionales y la empresa ya era fuente de trabajo de 20 familias aproximadamente.

A lo largo de ese año, se fueron incorporando más personas tanto al área de logística y soporte administrativo (BPO: Business Process Operations "Operaciones de procesos de negocios) como así también al área de desarrollo de software (KPO: Knowledge Process Operations - "Operaciones de procesos de conocimiento"), quedando conformada así por estas dos unidades de negocios. Actualmente el número de trabajadores asciende a 35 .

En un principio, los trabajadores de Nexius Córdoba, realizaban sus tareas en oficinas que la empresa poseía, con la posibilidad de trabajar desde sus hogares. Paulatinamente, y en vistas de que la gran mayoría elegía el método de trabajo homeworking y, además, con la idea de abaratar costos, se optó por que dichas oficinas dejasen de funcionar, prevaleciendo únicamente el trabajo remoto. Esta decisión fue un hito en la historia de la organización en la ciudad. Así, para el año 2009, todos los trabajadores de la sede ubicada en Córdoba pasaron a ser homeworkers, método que persiste hasta hoy en día.

\subsection{Misión y valores de la empresa}

La misión de Nexius se define en la siguiente frase: "Ser un socio confiable en la industria Wireless".

La meta de Nexius es convertirse en el socio confiable de los operadores de telefonía móvil a través de las soluciones (productos de software) que brinda. Es objetivo de Nexius coordinar a estos operadores a obtener sus propios objetivos, acoplan los objetivos de sus clientes con sus propias metas planteando, de esta manera, a Nexius como organización que se convierte como su aliado estratégico (Nexius. Recuperado el 10 de agosto, 2014, de http:/ / www.nexius.com/company).

Se consideran como valores de Nexius:

1. Satisfacción del cliente: el significado de este valor es definido como aquel que "enmarca aquellos sentimientos relacionados al cumplimiento de las expectativas que el cliente tiene sobre los productos y servicios de Nexius" (Nexius, ibidem).

2. Integridad: "ser-íntegro", como individuo íntegro, trasladado a una organización integra: aquella que se mueve dentro del mercado con ética y moral en sus acciones y decisiones organizacionales (Nexius, ibidem).

3. Innovación: es la introducción de "novedades en las acciones organizacionales" (Nexius, ibidem).

4. Trabajo en equipo: "No existiría otra forma de trabajo que no sea en equipo. Los procesos y fases que hacen a la producción, a la programación, al "testing" de los productos elaborados son siempre en equipo. No solo queda esta forma de trabajo como una metodología en sí, sino que también se traduce en un principio rector del comportamiento organizacional" (Nexius, ibidem). 


\subsection{Medios y acciones de comunica- ción}

Existen tres herramientas fundamentales a través de las cuales los empleados de Nexius Córdoba se mantienen conectados entre ellos. La principal y más utilizada es Skype, a través de la cual se realizan llamadas telefónicas, teleconferencias, etc. La empresa le otorga a cada empleado una cuenta para dicho programa, la cual posee ya de un crédito para poder realizar las llamadas telefónicas.

Otra herramienta muy utilizada es Outlook. Esto es simplemente un correo electrónico mediante el cual los empleados pueden compartir archivos, enviar información, etc.
Finalmente, los empleados de Nexius trabajan con un sistema operativo llamado Salesforce, dentro del cual está la posibilidad de hacer uso del "Nexius Neighbor". Este último es una página social donde cada empleado posee un perfil y, a través de ella cada uno puede compartir todo tipo de información, ya sea laboral como así también personal. De esta manera Nexius intenta generar un mayor conocimiento entre quienes trabajan en ella y, así, acercar más a los empleados entre ellos "disimulando" la distancia producto del contacto netamente virtual.

\section{Metodología}

El acercamiento a Nexius Córdoba se realizó desde una mirada cualitativa, llevando adelante un estudio de alcance exploratorio y parcialmente descriptivo. Esta forma de abordaje permitió, en primer lugar, familiarizarse con un tema novedoso para luego definir sus características, identificando sus propiedades, significados, usos, funciones $\mathrm{y}$ variaciones que en el tiempo/lugar y grupo social se presentaban (Emanuelli, 2009b).

Así, se determinaron cuatro variables de estudio que guían toda la indagación: estrategias comunicacionales, TIC, homeworking y sentido de pertenencia.

Estas variables permitieron el avance en el conocimiento del concepto homeworking como método de trabajo y su realidad empírica, caracterizar la influencia de las TIC en el proceso de comunicación interna de la empresa Nexius e identificar las características de las estrategias comunicacionales de la empresa aplicadas a sus empleados para desarrollar sentido de pertenencia.

En este marco, al observar que los datos y elementos que caracterizaban a la investigación son de carácter cualitativo (en tanto necesidad de determinar valores, creencias y visiones institucionales y personales) se decidieron utilizar dos herramientas: entrevistas semiestructuradas a empleados y análisis de contenidos de medios, productos gráficos digitales y acciones comunicacionales que la empresa utilizaba para generar sentido de pertenencia en sus trabajadores.

La elección de estas técnicas permitió, en lo que respecta a las entrevistas semiestructuradas, mantener un guión indirecto en la conversación e indagar en aquellos temas puntuales a la investigación, siempre posibilitando una amplitud en las declaraciones que logre profundizar en los campos interesados.

Por otro lado, relacionado con el análisis de contenido, permitió conocer la realidad del campo indagando en lo latente, en lo oculto dentro de los mensajes formales que la institución y los mismos empleados brindaban, generando categorías que den cuenta de esto.

\subsection{Elaboración de instrumentos y po- blación}

Las entrevistas a los empleados y el análisis de contendido de las mismas, junto con el análisis de los medios y acciones comunicacionales que la empresa utilizaba, mantuvieron una línea de coherencia con las cuatro variables establecidas, las cuales buscaban indagar elementos puntuales en cada una de ellas. 
En lo que respecta a las TIC se buscó saber sobre los medios, acciones o procedimientos de comunicación que disponen los empleados, cuáles prefieren, qué problemas presentan y su relación con la tecnología que utilizan.

Sobre el concepto de homeworking, cómo se sienten con este método de trabajo, las ventajas y desventajas que encuentran en relación al mismo, el tiempo y espacio de trabajo y su relación con los medios de comunicación.

Sobre el sentido de pertenencia se indagó qué conocimiento tienen los trabajadores acerca de la empresa, la relación de los homeworkers con sus pares y con la propia organización, la relación con su entorno personal a la hora de desempeñar sus tareas y la realización de reuniones formales e informales entre los trabajadores.

Por último, en las estrategias comunicacionales se indagó en cómo se desarrollan los procedimientos de comunicación, cómo se utilizan los medios y herramientas de comunicación que disponen los trabajadores para realizar sus tareas, qué acciones comunicativas lleva a cabo la empresa para hacerlos sentir parte, y el diagrama de un mapa comunicacional de la organización.

Las entrevistas se hicieron de forma aleatoria tratando de cubrir al menos un representante de cada área de Nexius Córdoba. El universo de los empleados que conforman el público interno de la empresa llega a las 35 personas, de los cuales se tomó una muestra de 10 miembros (ver Tabla 1).

Este número de entrevistas alcanzado en el procedimiento de muestreo permitió conseguir la saturación de las categorías analíticas planteadas en el estudio, por lo que no fue necesario continuar con la implementación de la metodología en más trabajadores.

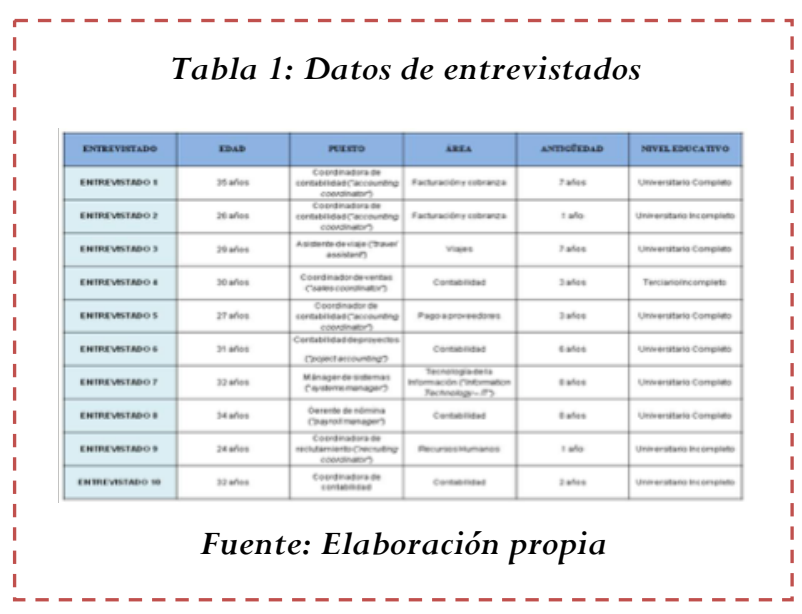

Las entrevistas fueron hechas durante la jornada laboral de los empleados. Por otro lado, cabe destacar que la totalidad de las entrevistas fueron realizadas vía Skype.

\section{Resultados y análisis de datos recolectados}

Siguiendo la lógica de las cuatro variables presentadas anteriormente, se muestran a continuación los resultados y el análisis de los datos recolectados con ambas herramientas.

\subsection{Estrategias comunicacionales}

En primera instancia, se afirma que existen interacciones igualitarias entre los trabajadores de Nexius Córdoba. Retomando los conceptos de Pasquali (1970), se observa a través de las entrevistas que tanto las relaciones sociales (tratos, lenguaje, códigos, etc.) como los medios utilizados por los homeworkers, generan una relación entre pares iguales, es decir, reciprocidad entre los interlocutores.

Otro dato importante que ayuda a mantener este concepto de "interacción igualitaria", es la política de ingreso por recomendación. En este sentido, se entiende que al permitir solo el ingreso de gente conocida, se logra mantener un perfil único dentro de la empresa el cual coincide con los valores de la misma ("Nexius Family"), generando homogeneidad entre los trabajadores e influyendo así en las relaciones entre los mismos. 
Un cambio observado en el proceso comunicacional que afectó a la empresa, fue la decisión de operar con la metodología homeworking en un cien por ciento, cerrando sus oficinas en las cuales algunos de sus actuales empleados trabajaban. A partir de esto, algunos trabajadores, pese a mostrar su afinidad con la totalidad de sus compañeros, evidenciaban su mayor cercanía con aquellos que compartieron dicho espacio físico. En este sentido, se confirma la idea de Pasquali (1970) en la que todo cambio en el proceso comunicacional (en este caso contexto y canal), afecta la relación entre los interlocutores (homeworkers). De esta manera, analizando los actores y medios, la posibilidad de generar procesos o contextos de comunicación cara a cara entre los empleados ayuda a fomentar relaciones más sólidas entre los mismos.

Por otro lado, tanto la posibilidad de hablar con lenguaje informal, como la política de recomendación para el ingreso generan igualdad tanto en las comunicaciones como en las relaciones. Las diferencias de estatus y roles organizacionales (en tanto parte del organigrama) no son condicionantes que generen diferencias relacionales. En cuanto a la forma en que la organización decide realizar sus tareas, se afirma que la política de trabajo en equipos favorece las interacciones igualitarias. Se reconocen divisiones internas de trabajos, cooperación entre los integrantes a la hora de tener que resolver problemas y una búsqueda de equilibrio entre las cargas laborales de cada uno. Al margen de identificar un líder en el grupo, los códigos, canales y modos de comunicación no varían por más que con éste se contacte. Por tanto, las relaciones entre pares se mantienen. Un punto a considerar es que los líderes de grupos, los gerentes, directivos y la misma cultura organizacional tienen como uno de sus objetivos generar este tipo de relaciones igualitarias, entre pares. Desde la idea de graficar una familia empresarial, la elección de medios de comunicación hasta formalizar el ingreso de nuevos miembros por recomendación, todo contribuye a generar un contexto de trabajo donde se lleven adelante comunicaciones igualitarias, de hacer más cercanas a las personas, donde los códigos y canales se mantienen independientemente del rol o estatus que se tenga dentro de la empresa (Pasquiali, 1970; Villada y Barroso 2006).

Por otro lado, observando las acciones comunicacionales, casi la totalidad de los empleados ha coincidido en que una acción con la que verdaderamente comienzan a sentirse parte de Nexius Córdoba, es cuando la misma les da la posibilidad de viajar a conocer la sede central de la empresa que se ubica en Texas, Estados Unidos. Comentan que al observar la forma en que se trabaja allá, interactuar con aquellas personas que solo conocían mediante un medio virtual, comienzan a darse cuenta del lugar al que pertenecen.

Otra acción que utiliza Nexius es hacer que el empleado sienta que el trabajo que realiza es realmente importante para la institución, mediante el reconocimiento explícito hacia el mismo (mensajes de textos, mails, mensajes en casilla de chatter, etc.), o bien indirecto comentando en las reuniones de grupo virtuales que se realizan diariamente. De esta forma, el empleado se esmera más para poder cumplir los objetivos.

Se destaca también ciertos after office que se realizan de manera espontánea, pero estos no son muy frecuentes. De igual manera, los entrevistados comentan que cuando se da esta posibilidad es bien aprovechada ya que de esta forma interactúan mejor con aquellas personas que trabajan todo el día.

Pensando así, los objetivos comunicacionales de la empresa serán brindar herramientas con las cuales los empleados puedan comunicarse eficientemente, respondiendo a las dinámicas de sus labores; que permitan suplir la comunicación informal, conectando a todos, independientemente de su status laboral, haciendo que el empleado se sienta parte de la institución, hacerle sentir que su labor vale, que es tenido en cuenta y es realmente importante para poder cumplir con los objetivos de Nexius.

Por otro lado, reina la idea de Nexius como una familia. Dado que las relaciones entre los empleados son netamente virtuales, buscan unir lazos entre ellos, que no sean simplemente empleados remotos sino que se desarrolle una relación de 
compañerismo, solidaridad, compromiso y, porque no, amistad. La imagen e idea de "familia" (nombrada dentro de la institución como Nexius Family) en este caso es estratégica y permite graficar eficientemente el modelo de relaciones comunicacionales que la empresa busca alcanzar, todo esto bajo la idea de que un empleado contento y con sentido de pertenencia trabaja mejor.

\subsubsection{Mapas comunicacionales}

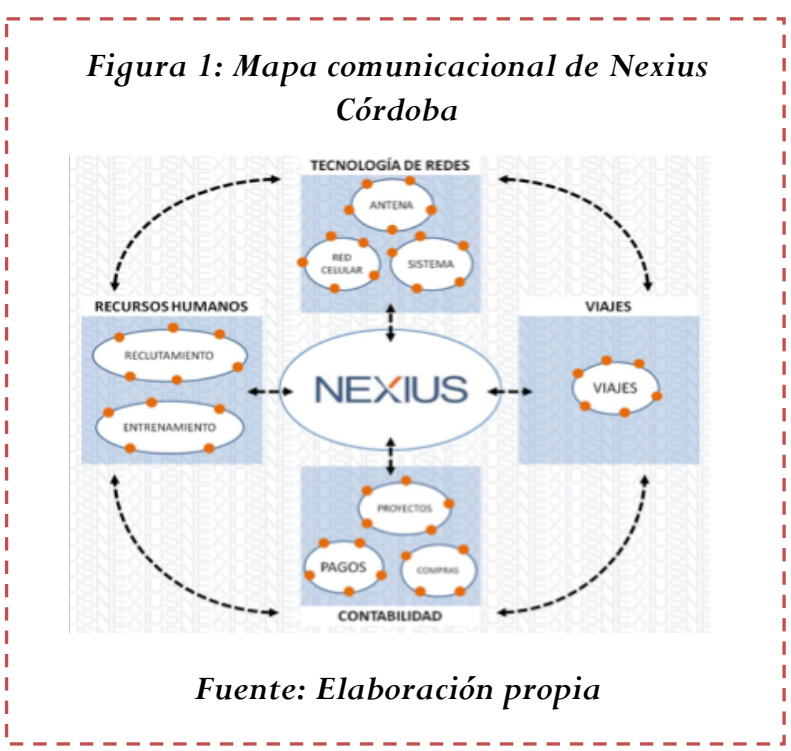

De este mapa (Figura 1) se pueden destacar varios aspectos. En primer lugar, su forma circular representa la idea de una comunicación horizontal, donde todos los empleados, indistintamente del cargo jerárquico, área o grupo de trabajo, pueden comunicarse entre sí sin ningún tipo de problema. Nadie es inalcanzable, la comunicación es muy fluida y de fácil acceso.

Se visualizan cuatro áreas fundamentales: Tecnología de Redes, Contabilidad, Viajes y Recursos Humanos. Cada una de ellas con sus respectivos grupos de trabajo e interconectadas de manera directa gracias a los medios y herramientas de comunicación anteriormente mencionados.

En lo que respecta a los grupos de trabajo dentro de dichas áreas, también son identificados con un formato circular. Aquí se da a entender un trato inmediato e igualitario entre sus empleados.
Esta horizontalidad graficada sustenta lo que algunos empleados señalaron acerca de la idea comunicacional que, en Nexius Córdoba, no existan brechas entre ellos, sino más bien se comportan como un todo integrado, como conjunto, complementados, es decir, buscando generar la comunicación de un grupo familiar (Nexius Family).

A partir de esto, se entiende que la estrategia comunicacional de Nexius Córdoba es, principalmente, intentar acercar a los empleados homeworkers entre sí, utilizando aquellos medios y herramientas que le son pertinentes, con el fin de generar esa "horizontalidad comunicacional" deseada. Esto, en vista de un planteo horizontal inicialmente propuesto desde las propias raíces de la cultura institucional.

\subsection{TIC}

Existen tres herramientas a través de las cuales los empleados de Nexius Córdoba se comunican entre ellos: Skype en primer lugar (la más utilizada), Outlook y un sistema operativo llamado Salesforce, dentro del cual está la posibilidad de hacer uso de un Chatter. Este último es una página social donde cada empleado posee un perfil y, a través de aquella, pueden compartir todo tipo de información, tanto laboral como personal.

A través del análisis de las entrevistas, se confirma que la elección de estos medios (por parte de la empresa) tiene coherencia con la relación social que se genera entre los empleados y la que pretende la empresa que sea.

La decisión de la compañía de brindar estas vías de comunicación a todos sus trabajadores está netamente vinculada con la idea de suscitar igualdad y reciprocidad entre ellos; y esto es lo que realmente sucede.

Los mismos no solo pueden verse y comunicarse sin diferenciación alguna, sino también, son capaces de visualizar la información de perfil de todos sus compañeros. 
Los medios de comunicación que utilizan están diseñados de tal manera que uno puede contactar a cualquier persona de la empresa de la misma manera y sin restricciones. Las divisiones de contactos (o formación de grupos) que se pueden realizar son opciones que cada usuario tiene y es solo parte de la organización personal del mismo. Los programas en sí no generan divisiones o restricciones a contactos, todos son visibles, se les accede de la misma manera y están alistados en el mismo sector y lugar.

En otras palabras, se observa una lógica coherente entre las relaciones que se desarrollan (igualitarias, de pares), aquellas que busca generar la empresa en función de sus valores y los TIC que se utilizan.

Se confirma de este modo el elemento social de la tecnología utilizada (Castells, 2009), su influencia en las relaciones y su lógica con los valores/políticas institucionales.

Por otro lado, se afirma que la empresa ha amoldado la tecnología al objetivo, cultura y políticas institucionales, haciendo un uso inteligente de las TIC (Sessa V. et al. 1999). Esto se entiende ya que, basado en las entrevistas, los usos que permiten las herramientas comunicacionales brindadas por la organización tienen coherencia con los valores y políticas expresadas por los empleados.

Por otro lado, es unánime la aceptación de las TIC seleccionadas por la empresa para cumplir los objetivos laborales y las funciones comunicacionales de los empleados. Esta relación se afirma positiva, dando cuenta un cabal entendimiento de las herramientas por parte de los empleados y su utilización concreta en la actividad. Los homeworkers hacen ver su conocimiento sobre los medios de comunicación, sabiendo discriminarlos en función del mensaje a enviar y el destinatario deseado.

\subsection{Homeworking}

Apoyados en los autores de "Geographically Dispersed Teams: an annotated bibliography", se entiende que la dinámica de los homeworking de Nexius Córdoba se basa en dos relaciones: de diferente tiempo y diferente espacio; y mismo tiempo y diferente espacio. Para la primera combinación, se tienen en cuenta las comunicaciones que tienen los empleados con sus compañeros de Estados Unidos. En este caso, los homeworking se encuentran en diferentes márgenes horarios y en diferentes lugares físicos de trabajo. La comunicación mediada será así atravesada por esta contingencia. En el segundo caso (mismo tiempo diferente espacio) se circunscriben las relaciones entre los homeworking de Nexius Córdoba. Estos mantienen un mismo sentido horario, pero no así un mismo lugar de trabajo.

Por otro lado, la relación con el método es eficiente y positivo, basándose en la selección de los recursos humanos y la solución a las desventajas que da cuenta dicho método.

Afirmando la buena relación entre los trabajadores y el formato de trabajo, uno de los puntos importantes es la selección de los líderes de trabajo para que esto pueda desarrollarse. Se afirma que el reconocimiento al trabajo del homeworker por parte de los líderes existe, la capacidad de liderar democráticamente, mantener enfocados a los empleados en los objetivos a alanzar y generar confianza entre los miembros de grupo. Al decir líderes cabe aclarar que dentro de la cultura informal de la empresa, estos actores se muestran cercanos, pares en relación con sus compañeros. Este elemento también genera una mejor predisposición de los empleados hacia el método de trabajo.

Por otro lado, la buena selección de los propios homeworkers es también un punto a tener en cuenta. Se entiende que la política de ingreso por recomendación ayudó a que los empleados coincidan en un perfil acorde al método de trabajo, manteniendo márgenes de edad jóvenes, con manejo y desarrollo mediante las TIC, y capacidad para motivarse trabajando eficientemente de manera individual (Sessa V. et al., 1999).

Los mismos también dan cuenta de las ventajas y desventajas que el homeworking tienen para los empleados y la empresa, y así mismo generan soluciones creativas a la ausencia de contacto personal, el establecimiento poco claro de la vida personal y laboral, la comunicación poco continua 
entre compañeros y la necesidad de autodisciplina y organización (Conferencia ADHRA, 2013).

\subsection{Sentido de pertenencia}

Habiendo realizado las entrevistas, se pudo dar cuenta que los empleados poseen un conocimiento general acerca de las tareas que se desarrollan en Nexius Córdoba. Sin embargo, cada uno de ellos tiene una noción más específica de acuerdo al área en la cual desempeña sus actividades.

Otro dato importante es que, en función de la antigüedad del empleado, se pudo constatar un mayor o menor conocimiento sobre los trabajos que la empresa realiza, como así también de las actividades que las demás áreas llevan a cabo. Esto se repitió, incluso, cuando se indagó acerca de la cultura institucional (valores, misión y visión). Es decir, a mayor antigüedad, mayor conocimiento.

Por otra parte, se vislumbró que los homeworkers no reconocen los valores formales que la empresa promulga, sino que, por el contrario, se encuentran más familiarizados con aquellos informales, relacionados con el día a día de su actividad. Se afirma así que el conocimiento que los empleados tienen de la empresa se circunscribe más a sus áreas de trabajo y la actividad interna de sus equipos, que al general de la organización. En otras palabras, lo informal o instituyente, aquellos elementos que no están formalizados en algún documento institucional son aquellos que predominan en el conocimiento de los empleados sobre la empresa (García Jiménez, 1998).

Siguiendo el análisis, aquellos empleados que supieron trabajar en las oficinas de la empresa (antes de pasar a ser solo homeworkers), acreditan mayor conocimiento general de la organización, ya que pudieron relacionarse con compañeros de diferentes áreas y así ampliar su entendimiento sobre las acciones de Nexius. En tanto, el bagaje se incrementa por canales informales (experiencias y relaciones con otros empleados), no así por un medio formal de comunicación.
Por otro lado, se puede afirmar que la relación entre los empleados de Nexius Córdoba es muy buena. Predomina una idea de compañerismo, reciprocidad y solidaridad, lo cual genera un buen clima laboral. En otras palabras, se reconocen lazos de afiliación (amistad, apoyo moral e identificación) con los grupos de trabajo (Schein, 1982).

Un primer punto a considerar es que entre aquellos trabajadores que compartieron las oficinas de la empresa se observa una mayor afiliación basada en aquella experiencia. Se identifican lingüísticamente, se sienten parte de un grupo informal y son reconocidos por aquellos que ingresaron luego de la reestructuración. Así, las experiencias compartidas y el tiempo en la empresa fortalecen los lazos de amistad, la afiliación al grupo y la organización, permitiendo mantener el clima positivo en el contexto de trabajo. Estos modos se transmiten hacia aquellos trabajadores más jóvenes de la institución $\mathrm{y}$, junto con lo expuesto en el punto relacionado a las Estrategias Comunicacionales, se logra una coherencia que concluye en relaciones positivas e igualitarias (García Jiménez, 1998). En este sentido, se reconoce la importancia que le dan todos los empleados a las instancias de comunicación cara a cara, las cuales favorecen contundentemente al fortalecimiento del sentido de pertenencia de los actuales trabajadores.

Por otro lado, la afiliación se da en su gran mayoría dentro de los grupos formales de trabajo. Es decir, al contar con poco contacto (o directamente no tenerlo) con otras áreas o equipos de trabajo, los grupos laborales más directos forman sus lazos de contención y se circunscriben en sí mismos. Teniendo en cuenta casos de excepción, son los mismos grupos formales las bases desde donde se generan los grupos informales, siendo estos últimos los que permiten los lazos de amistad, compañerismo, reciprocidad y afiliación. Así, las acciones comunicativas de los empleados circunscriptas solo a su grupo de trabajo directo repercuten en la formación de lazos personales con sus compañeros y el sentido de pertenencia hacia la empresa. 
Otro punto es la política de ingreso por recomendación. En este caso, los trabajadores ingresan con algún lazo de amistad previa al trabajo, lo cual permite una mayor comodidad al grupo laboral y al individuo que se suma. La afiliación se facilita ya que se adelantan ciertos pasos a la hora de conocerse y se tienen referencias más cercanas de ambas partes. La familiaridad con el empleado es más concreta, haciendo que la inserción al grupo de trabajo se acelere.

Se puede afirmar entonces que existen en la empresa marcados lazos de afiliación entre los trabajadores que comparten un mismo grupo de trabajo. Estos se acrecientan mientras más experiencias compartidas vivan los empleados, siendo puntos de apoyo importantes la política de ingreso por recomendación $\mathrm{y}$, contundentemente, las instancias de comunicación cara a cara que estos grupos de trabajo puedan compartir.

Continuando la misma lógica, el fenómeno se repite para el sentido de pertenencia que los empleados tienen hacia la empresa. La relación es positiva, y se reconocen mayores corporizaciones en aquellos empleados que lograron experimentar momentos de relación directa (cara a cara) con distintos compañeros, directivos o gerentes; o bien con lugares físicos de la empresa, tanto en las oficinas que la organización supo tener en Córdoba o viajando a otras ciudades donde ésta se radica.

Así bien, la relación con la empresa por parte de los homeworkers inicia su camino en los lazos que se forman dentro de los grupos de trabajo más directos. Las experiencias que en estos se obtienen son las vías de transmisión de los valores, creencias, objetivos, mitos, hábitos y normas institucionales más representativas. Aun siendo cercanos a los objetivos, valores, visión y misión institucionalizada, estos elementos culturales transmitidos son de carácter informal y se alteran dependiendo del trabajador que responde. Por tanto, no se encuentra un conocimiento cabal del elemento cultural formal de la empresa, una respuesta uniforme que reproduzca los elementos culturales instituidos. Así bien, se afirma que la cultura informal expuesta por los homeworkers es funcional y eficiente para el funcionamiento de los grupos de trabajo y el alcance de los objetivos empresariales, lo cual da cuenta de una buena transmisión y entendimiento de la lógica laboral por parte de los empleados.

\section{Discusión}

La primera cuestión que se plantea es hacia la idea de primacía de las relaciones mediatizadas y el uso de las TIC. En este sentido, la investigación nos afirma que (en una universalización) para generar sentido de pertenencia en trabajadores totalmente relacionados por las TIC, es clave en la estrategia comunicacional generar momentos de comunicación directa (cara a cara). Por tanto, se crean incógnitas que bien sirven para reflexionar sobre el hecho tecnológico y su función real dentro de la comunicación organizacional: ¿Cuáles son los sus límites? ¿Qué medios/acciones se priorizarán a la hora de generar grupos de trabajo? ¿Cómo se plantearán las políticas comunicacionales de las organizaciones? Sea como fuese, la comunicación directa (cara a cara) mostró que, en un mundo mediatizado, no podrá dejarse de lado, e incluso más, debe ser una política comunicacional estratégica a la hora de planificar nuestros objetivos.

La segunda discusión apunta a que, de una u otra manera, es imposible evadir el avance tecnológico y será necesario generar medios para desarrollar afiliaciones, buenos climas laborales y sinergias de trabajo (que posibiliten el alcance de los objetivos comunicacionales) utilizando comunicaciones mediatizadas. Inclusive, por diferentes hechos sociales y culturales se generan cambios estructurales en la vida cotidiana de los individuos que hacen que, ellos mismos, elijan metodologías de trabajo flexibles como el homeworking. Por tanto, si la tendencia es generar más movilidad, flexibilidad e inmediatez comunicacional al trabajador, se observa en el estudio, será el desarrollo y elección minuciosa de las herramientas tecnológicas el factor 
que marque la diferencia, lo que posibilitará alcanzar los objetivos organizacionales y, a su vez, los personales de cada empleado.

Esto se afirma con la mirada cualitativa con la que se abordó el estudio, donde se analizaron las particularidades del caso y a sus agentes. Esta forma de abordaje cualitativo fue acorde al objetivo propuesto, logrando obtener validez en los datos y, a su vez, se recomienda replicar en futuros estudios que tengan relación con el tema.

Como limitaciones se puntualiza el hecho de trabajar sobre elementos tecnológicos. En este sentido, los avances son veloces y surgen variedad de soluciones que se aceptan culturalmente (y en otros casos se exigen) para todo inconveniente comunicacional. Lo cual hace que los estudios se vean en constante revisión.

\section{Conclusiones}

Respondiendo a la pregunta de investigación planteada (¿cuáles son las estrategias comunicacionales que permiten desarrollar el sentido de pertenencia en trabajadores bajo el método homeworking?), se llega a la conclusión de que existen dos variables fundamentales que dan solución a la incógnita: a) la confirmación de las relaciones directas (cara a cara) como herramientas comunicacionales estratégicas a la hora de generar sentido de pertenencia en una organización donde las relaciones son completamente mediadas; y b) la importancia de contar con una elección y utilización racional de las TIC.
Ambas variables se erigen sobre una misma base: trabajar siempre bajo un diagnóstico exhaustivo antes de planificar la comunicación organizacional. En este caso fue cualitativo, lo cual se recomienda en este tiempo de estudios.

De esta manera, se asevera la necesidad continua de generar estudios y seguir brindando información de relevancia en este campo comunicacional que se relaciona con las TIC, ya que el avance de estas es permanente y las teorías disponibles siempre serán adelantadas por una nueva tecnología, aplicación o dispositivo. Por tanto, se alienta a la continuidad de las prácticas de investigación, a la apertura de los campos, buscando ser el presente un pequeño aporte a la causa.

\section{Referencias}

- Alles, M (1999). Empleo: discriminación, teletrabajo y otras temáticas. Ed. Macchi.

- Álvarez Nobell, A. Romero Calmache, M. Sánchez Sanvicente, M. Aragüés Dufol, P. (2012). "Desarrollo cultural en las organizaciones. Un modelo de estudio basado en la "Grounded Theory”. Revista Mediterránea de Comunicación, vol. $3, \mathrm{n}^{\mathrm{o}} .2$, 111-127. Recuperado de http://www.rmedcom.org/2012/1211/121 1Alvarez_es.htm.

- Bellomo, A. (2013). "En nuestro país el teletrabajo está creciendo de manera agigantada, calculándose más de 1800000 oficinas en el hogar". Recuperado enero 15,
2013, de Eleve Sitio web: http://www.eleve.com.ar/En-nuestro-pais-el-teletrabajoesta-creciendo-de-manera-agigantadacalculandose-mas-de-1800000-oficinas-en-elhogar.html

- Castells, M (2009). La Era de la Información. La sociedad Red. Tomo 1. Ed. Alianza

- Emanuelli, Paulina B. (2009b). Herramientas de Metodología para investigar en comunicación. Tomo II. Córdoba, Argentina.

- García Jiménez, J. (1998). La Comunicación Interna. Madrid, España: Díaz de Santos

- Lenguita, P. (2005). Teletrabajo: El impacto de las tecnologías informáticas y comunicacionales 
sobre el empleo. Ministerio de trabajo de la Provincia de Buenos Aires.

- Nexius. Recuperado el 10 de agosto, 2014, de http://www.nexius.com/company.

- Pasquali, A (1970). Comprender la comunicación. Ed. GEDISA

- Schein, E. (1982). Psicología de la Organización. Prestice Hall.

- Scheinsohn, D. (2009). Más allá de la Imagen Corporativa. Cómo crear valor a través de la Comunicación Estratégica. Ed. Macchi.
- Sessa Valerie I.; Hansen Michael C.; Prestridge Sonya y Kossler Michael E. (1999). Geographically Dispersed Teams: an annotated bibliography. Forming, developing and maintaining GDTs. Center for creative leadership.

- Villada, M.L., Barroso, C. (2006). El proceso de comunicación interna en un equipo virtual de trabajo. Trabajo Final de Grado.

\section{Forma de Citación}

COELHO, Nicolas Joel: Homeworking: Estrategias comunicacionales y sentido de pertenencia. Revista Communication Papers, $\mathrm{N}^{\circ}$ 7, páginas 96 a 108. Departamento de Filología y Comunicación de la Universidad de Girona. Recuperado el__ de de 2 de: http://www.communicationpapers.es 